\title{
Simple Mechanisms to Implement the Core of College Admissions Problems*
}

\author{
José Alcalde \\ Departamentas Fonaments de l'Anàlisi Econòmica, University of Alicante, \\ E-03071 Alicante, Spain \\ and

\begin{abstract}
Antonio Romero-Medina
Department of Economics, Universidad Carlos III de Madrid, 28903 Getafe, Spain
\end{abstract}

\begin{abstract}
This paper analyzes simple mechanisms implementing (subselections of) the core correspondence of matching markets. We provide a sequential mechanism which mimics a matching procedure for many-to-one real life matching markets. We show that only core allocations should be attained when agents act strategically when faced with this mechanism. We also provide a second mechanism to implement the core correspondence in Subgame Perfect Equilibrium. Journal of Economic Literature Classification Numbers: C78, D78.
\end{abstract}

\section{INTRODUCTION}

In this paper we introduce two mechanisms that implement the stable correspondence in a college admissions problem. These are stylized versions of contractual processes in bilateral markets where monetary transfers either are irrelevant or can be embodied on agents' preferences.

*We thank Jabier Arin, Salvador Barberà, Luis Corchón, Carmen Herrero, Iñigo IturbeOrmaetxe, Matthew O. Jackson, Martin Peitz, Socorro Puy, Tayfun Sönmez, and an associate editor for their comments. Alcalde's work is partially supported by the Institut Valencià d'Investigacions Econòmiques and DGICYT under project PB 97-0131. Romero-Medina acknowledges financial support from the Instituto de Estudios Fiscales and DGICYT under project PB 92-0590. 
Matching markets have been extensively analyzed from a gametheoretical point of view (see Roth and Sotomayor (1990) for a detailed state of the art until 1990). In this framework, Roth (1982) and Alcalde and Barberà (1994) have shown the existence of incentives for agents to misreport their true preferences. These incentives appear when agents are faced with some mechanisms selecting allocations that satisfy certain "desired" properties. These results give us a reason to study the implementability of stable solutions for the college admissions problem.

Core correspondence implementability in college admissions problems was first approached by Kara and Sönmez (1997). They showed that the core correspondence can be implemented in Nash equilibrium. However, they do not provide a simple mechanism that can be used in real-life situations. They also show that no subselection of the core is Nash implementable. There are two problems that are still open in the college admissions problems framework. The first one is the design of natural mechanisms to solve the implementability of the core, and the second one is the analysis of implementability of core subselections. This paper provides positive answers to both of them.

Alcalde (1996) studied both questions for a particular case of matching problems, the marriage market problem. He provides some positive answers to the implementation problem, using simple mechanisms. In particular, he implements the core correspondence. He also provides a mechanism to implement the extreme points in the core correspondence.

A way to deal with the problem is to analyze simple real-life mechanisms. Romero-Medina (1998) studies the mechanism employed by the Spanish university system to allocate new students to colleges. He shows that this mechanism can select unstable outcomes, while core allocations would be reached when students behave strategically. This result cannot be applied to the more general framework in which we are interested, since the matching procedure studied in Romero-Medina (1998) does not allow universities to behave strategically.

Let us now introduce the mechanism that implements the core correspondence in bilateral matching markets. It is a procedure in which agents take decisions sequentially. In the first stage, students simultaneously send an application form to no more than one college. In the second stage, each college selects its best set of students from among the applicants. Colleges' decisions determine the matching which results from agents' interaction. Thus, the mechanism to be analyzed captures essential aspects which hold in real-life college admissions problems. First, we model sequential interactions among agents on both sides of the market, reflecting an adjustment process to reach stable allocations. Second, agents on one side of the market (students) adopt an "active" role, making offers, whereas the aptitude shown by agents on the other side (colleges) can be considered as "passive": 
they only accept or reject the offers they receive. In fact, the mechanisms analyzed below reflect the idea of the classical algorithm developed by Gale and Shapley (1962).

Since our mechanism reflects a sequential decision process, the first equilibrium concept we study is Subgame Perfect Nash Equilibrium (SPE). Therefore, our results cannot be seen as a consequence of those obtained by Kara and Sönmez (1997). We are also interested in analyzing the effects of the agents' commitments when selecting the strategies to be played. Thus, we also analyze the outcomes that can be expected when agents select strategies that constitute a Strong Subgame Perfect Nash Equilibrium (SSPE) for this game, that is, strategies that are not only a SPE but also a Strong Nash Equilibrium for this mechanism. Under such an equilibrium concept, this mechanism selects the students' optimal stable matching. This matching is the (unique) matching weakly preferred by every student to any other stable matching.

Notice that the main feature of the mechanisms that we present in this paper is that they constitute reasonable proposals for effective design. Following Jackson (1992), the mechanisms used to implement social choice correspondences should have "natural" features. Applicability to real-life situations can be one of these features. The mechanism we present in this paper satisfies this requirement, and furthermore it is traditionally used in many real-life situations. There are two main reasons for this: first, it is straightforward to obtain the message space of each agent from its own preferences. Second, any individual is able to evaluate the consequences of her strategy without using a sophisticated analysis of the mechanism.

The rest of the paper is organized as follows. Section 2 introduces the basic model. Section 3 presents and analyzes the mechanism, to be called "the students-propose-and-colleges-choose" mechanism. Section 4 studies the effects of an interchange in agents' roles in the mechanism introduced in Section 3. Conclusions are presented in Section 5.

\section{THE MODEL}

Consider a college admission problem with $n$ colleges and $m$ students. Let $C=\left\{c_{1}, \ldots, c_{n}\right\}$ and $S=\left\{s_{1}, \ldots, s_{m}\right\}$ be the sets of colleges and students, respectively. Each college has preferences, $P(c)$, of sets of students. $P(c)$ is assumed to be a linear order on $2^{S}$. Each student's preferences, $P(s)$, is described by a linear order on $C \cup\{s\}$. A college admissions problem is fully described by a triplet $\{C, S ; P\}$, where $P=$ $\left\{P\left(c_{1}\right), \ldots, P\left(c_{n}\right), P\left(s_{1}\right), \ldots, P\left(s_{m}\right)\right\}$ is a list containing a full description of the agents' preferences and is called a profile. 
An allocation for such a problem, or matching, is a mapping $\mu$ from $C \cup S$ into $2^{S} \cup C$ satisfying

(i) For all $c \in C, \mu(c) \in 2^{S}$.

(ii) For all $s \in S, \mu(s) \in C \cup\{s\}$.

(iii) For each pair $(c, s) \in C \times S,[\mu(s)=c \Longleftrightarrow s \in \mu(c)]$.

From now on we will consider $C$ and $S$ to be fixed sets. Thus we can identify a college admissions problem $\{C, S ; P\}$ with the preference profile $P .{ }^{1}$ Let $M$ be the set of all possible matchings $\mu$. Finally, $\mathbb{P}$ denotes the set of (potential) matching markets.

Let $P$ be a matching market. Given a set of students $A \subseteq S$, we denote by $\left.C h_{c} \widetilde{(} A\right)$ the maximal element on $2^{A}$ under the linear order $P(c)$.

Definition 2.1. A matching $\mu$ is said to be individually rational for $P$ iff

(i) $C h_{c}(\mu(c))=\mu(c)$ for all $c \in C$.

(ii) For all $s \in S, c \in C[s P(s) c \Longrightarrow s \notin \mu(c)]$.

Definition 2.2. Let $\mu$ be a matching for $P$. We say that $\mu$ is blocked by a pair $(c, s) \in C \times S$ iff

(i) $c P(s) \mu(s)$.

(ii) $s \in C h_{c}(\mu(c) \cup\{s\})$.

A pair $(c, s)$ which satisfies the above two conditions is called a blocking pair for $\mu$.

Definition 2.3. Let $\mu$ be a matching for $P$. We say that $\mu$ is (pairwise) stable if it is individually rational and there is no pair blocking it. Let $\mathcal{N}(\underset{\sim}{P})$ denote the set of stable allocations for the problem $\underset{\sim}{P}$.

Finally, we assume that colleges' preferences, regarding groups of students, are substitutive. That is, for any two students $s \neq s^{\prime}$, if $s$ belongs to $C h_{c}(A)$, then she will also belong to $C h_{c}\left(A \backslash\left\{s^{\prime}\right\}\right)$. This is an usual assumption in the related literature, and it guarantees nonemptiness of the set of stable allocations (see Theorem 6.5 in Roth and Sotomayor (1990)). Notice that when colleges' preferences are substitutive, the set of (pairwise) stable allocations coincides with the core of that college admissions problem. $^{2}$ That is, given a stable allocation, no group of agents can find a

${ }^{1}$ For the sake of simplicity, we use the same notation for a preference profile and for the related college admissions problem. The context will precise if $P$ denotes a matching problem or simply a preference profile.

${ }^{2}$ Proposition 6.4 in Roth and Sotomayor (1990) establishes that stability and pairwise stability are equivalent concepts in college admissions problems with substitutive preferences. 
matching to improve the utility of all of its members without being matched with agents outside this group. Furthermore, if colleges' preferences satisfy substitutability, the set of stable allocations has a latticial structure. This property guarantees (i) the existence of a unique stable allocation which is Pareto optimal from the point of view of students and (ii) the existence of a unique allocation which is Pareto optimal from the point of view of colleges (when restricted to the set of stable matchings).

The concept of implementation used throughout the paper is well known in the literature. We next formalize this concept for both the Subgame Perfect Nash Equilibrium (SPE) and the Strong Subgame Perfect Nash Equilibrium (SSPE) cases. Let $\mathscr{E}_{k}$ be the set of strategies for agent $k$, and let $\mathscr{E}=\times_{x \in C \cup S} \mathscr{E}_{x}$ be the set of strategy profiles. Associated with each strategy profile $\tilde{e} \in \mathscr{E}$ we can define a message profile $m(\tilde{e})$, or simply $\tilde{m}$, which describes the action taken by each individual, given the strategy they choose. A matching mechanism is described by the set of strategies available to each agent and an outcome function $\gamma$ that assigns a matching to each profile of messages. We say that a matching mechanism implements a solution concept, say $\chi$, in (Strong) Subgame Perfect Nash Equilibrium if (i) for any $\tilde{e}$, (Strong) Subgame Perfect Equilibrium of the game $\Gamma:=\{C, S ; P ; \gamma\}$, $\gamma(m(\tilde{e}))$ belongs to $\chi(\underset{\sim}{P})$, and (ii) for each $\mu$ in $\chi(\underset{\sim}{P})$ there exists a (Strong) SPE for $\Gamma$, say $\tilde{\tilde{e}}^{\prime}$, such that $\gamma\left(m\left(\tilde{e}^{\prime}\right)\right)=\mu$.

\section{THE "STUDENTS-PROPOSE-AND-COLLEGES- CHOOSE" MECHANISM}

This section is devoted to analyzing a matching mechanism that mimics real-life matching procedures. In the mechanism we propose, each student selects the college at which she wants to study. Then, once each college had received all of its application forms, it accepts its most preferred set of students.

Let us introduce the mechanism, which we are going to call the studentspropose-and-colleges-choose mechanism. This is a two-stage game. In the first stage, students have to decide. Each student's message space coincides with the set of colleges and her being unmatched, $C \cup\{s\}$. In the second stage colleges, knowing students' messages, select the set of students that they want to admit. Thus, each college's message space coincides with $2^{S}$. Let $m(k)$ denote the message of agent $k \in C \cup S$, and let $\tilde{m}$ be an ordered vector containing the messages of all of the agents.

The outcome function, denoted by $\Phi^{S C}$, selects a matching which is defined as follows: $\Phi^{S C}(\tilde{m})=\mu_{\tilde{m}}$, where for any $s$ in $S$,

$$
\mu_{\tilde{m}}(s)= \begin{cases}m(s) & \text { if } s \in m(m(s)) \\ s & \text { otherwise }\end{cases}
$$


and, for each $c$ in $C$,

$$
\mu_{\tilde{m}}(c)=\{s \in m(c) \mid c=m(s)\} .
$$

THEOREM 3.1. The "students-propose-and-colleges-choose" mechanism implements in SPE the core correspondence of college admissions problems.

Proof. First, we show that every SPE outcome is a stable matching relative to agents' preferences. Let $\tilde{m}^{\prime}$ be a SPE for $\Gamma^{S C}:=\left\{C, S ; P ; \Phi^{S C}\right\}$. One can check that, at the second stage, each college has a dominant strategy, namely, $m^{\prime}(c)=C h_{c}\left(\left\{s \in S \mid c=m^{\prime}(s)\right\}\right) .{ }^{3}$ Thus, $\Phi^{S C}\left(\tilde{m}^{\prime}\right)$ should be an individually rational matching for $P$.

Let us suppose that $\Phi^{S C}\left(\tilde{m}^{\prime}\right)$ is not in $\mathcal{N}(P)$; then there should be a blocking pair, say $(c, s)$, in $C \times S$. Since all of the students play simultaneously, this cannot be the case, because student $s$ can reach higher utility by playing $m^{\prime \prime}(s)=c$. Notice that, in the second stage, $c$ 's message has to contain such a student, a contradiction.

On the other hand, let $\mu$ be a stable matching for $P$. Let us consider the following strategies for the agents. Each student message (and strategy) is $m(s)=\mu(s)$. In the second stage any college's strategy is its dominant strategy, its message being $m(c)=\mu(c)$. This constitutes a SPE for the related game whose outcome coincides with $\mu$, which yields the desired result.

Since the Social Choice Correspondence that we study is the core, we analyze the influence of agents' behavior on the expected outcome when commitment is allowed. In such a case Strong Subgame Nash Equilibrium seems to be a minimum requirement to be fulfilled by our predictions. The analysis of such an equilibrium concept is the aim of Theorem 3.2.

THEOREM 3.2. The "students-propose-and-colleges-choose" mechanism implements in SSPE the students optimal stable allocation.

Proof. First, we are going to show that the students' optimal stable matching can be supported by a SSPE. Let $P$ be a matching market, and let $\mu^{S}$ be its students' optimal stable allocation. Consider the following strategies: for any $s$ in $S, m(s)=\mu^{S}(s)$, and, for each $c$ in $C, m(c)=\arg \max P(c)$ on $\{s \in S$ s.t. $c=m(s)\}$. It is straightforward to see that these strategies constitute a SSPE whose outcome is $\mu^{S}$.

\footnotetext{
${ }^{3}$ Notice that such a strategy is not its unique best response. In fact, a necessary and sufficient condition for $m(c)$ to be a college $c$ 's best response to students' strategies, $m(s)$, is to satisfy $m(c) \cap\{s \in S \mid c=m(s)\}=C h_{c}\left(\left\{s \in S \mid c=m^{\prime}(s)\right\}\right)$. Nevertheless, all of these messages are strategically equivalent. Since we are interested in equilibrium payoffs rather than equilibrium strategies, we do not pay attention to these strategies.
} 
On the other hand, let $\tilde{m}^{\prime}$ be a SSPE yielding $\mu \neq \mu^{S}$ as the outcome. We will show that it is not possible. Notice that every SSPE is a SPE. Thus, by Theorem 3.1, $\mu$ has to be stable. Let denote $S^{\prime}:=\left\{s \in S: \mu^{S}(s) P(s) \mu(s)\right\}$ the set of students preferring their allocation under $\mu^{S}$ rather than under $\mu$. Since $\mu \neq \mu^{S}, S^{\prime}$ is nonempty. Now consider the following strategies: every $s$ in $S^{\prime}$ plays $m^{\prime \prime}(s)=\mu^{S}(s)$, and any $s$ in $S \backslash S^{\prime}$ plays $m^{\prime}(s)$. Following the lattice structure of the core, it holds that $m^{\prime}(s)=\mu^{S}(s)$ for all $s$ not in $S^{\prime}$. Given that colleges play their dominant strategies (see the proof of Theorem 3.1 above), the outcome when agents in $S^{\prime}$ shift their strategy and play $m^{\prime \prime}(s)$ is $\mu^{S}$, a contradiction.

\section{THE "COLLEGES-PROPOSE-AND-STUDENTS- CHOOSE" MECHANISM}

This section introduces a mechanism implementing the core correspondence of college admissions problems. The idea underlying this mechanism is very similar to the one analyzed in Section 3. In this case offers are made by colleges, and each student selects her "best college" from the proposals she receives. That is, the main formal difference between this mechanism and the one studied in Section 3 is that we shift the order in which agents on both sides of the market make their decisions.

A formal description of the mechanism, named "the colleges-proposeand-students-choose" mechanism, follows. It is a two-stage game. In the first stage, colleges have to decide simultaneously. Each college message space coincides with the set of potential teams of students, $2^{S}$. In the second stage, students, knowing colleges' messages, select simultaneously the college in which they want to study. Thus, each student message space coincides with $C \cup\{s\}$. Let $m(k)$ denote the message by agent $k \in C \cup S$, and let $\tilde{m}$ be an ordered vector containing agents' messages.

The outcome function, denoted by $\Phi^{C S}$, selects a matching which is defined as follows: $\Phi^{C S}(\tilde{m})=\mu_{\tilde{m}}$, where for any $s$ in $S$,

$$
\mu_{\tilde{m}}(s)= \begin{cases}m(s) & \text { if } s \in m(m(s)) \\ s & \text { otherwise }\end{cases}
$$

and, for each $c$ in $C$,

$$
\mu_{\tilde{m}}(c)=\{s \in m(c) \mid c=m(s)\} .
$$

The next result analyzes the equilibria outcomes of this mechanism when no commitment by agents is allowed. In some sense, the result can be interpreted as an equivalence between this mechanism and the "studentspropose-and-colleges-choose" mechanism. The proof for Theorem 4.1 is omitted, but it can be constructed in a way similar to that of Theorem 3.1. 
THEOREM 4.1. The mechanism described above implements in SPE the core correspondence.

The relationship found between the mechanisms studied in Theorems 3.1 and 4.1 does not hold when agents are allowed to commit on deciding which strategies have to be played. As Example 4.2 shows, cooperation among agents does not necessarily reduce the set of possible outcomes.

EXAMPLE 4.2. Let us consider the following five students and three colleges market:

$$
\begin{array}{ll}
P\left(s_{1}\right)=c_{1} & P\left(c_{1}\right)=s_{1} s_{2} s_{3} s_{4} s_{5} \\
P\left(s_{2}\right)=c_{3} c_{1} c_{2} & P\left(c_{2}\right)=\left(s_{2} s_{3}\right)\left(s_{4} s_{5}\right) s_{1} s_{2} s_{3} s_{4} s_{5} \\
P\left(s_{3}\right)=c_{3} c_{1} c_{2} & P\left(c_{3}\right)=\left(s_{4} s_{5}\right)\left(s_{2} s_{3}\right) s_{1} s_{2} s_{3} s_{4} s_{5} \\
P\left(s_{4}\right)=c_{2} c_{1} c_{3} & \\
P\left(s_{5}\right)=c_{2} c_{1} c_{3} &
\end{array}
$$

It is straightforward to see that there is a Strong Subgame Perfect Nash Equilibrium yielding each stable matching. For instance, the matching $\mu^{S}$ in which $\mu^{S}\left(c_{1}\right)=s_{1}, \mu^{S}\left(c_{2}\right)=\left(s_{4} s_{5}\right)$ and $\mu^{S}\left(c_{3}\right)=\left(s_{2} s_{3}\right)$ can be supported in SSPE by strategies $m\left(c_{1}\right)=\left(s_{1} s_{2} s_{3} s_{4} s_{5}\right), m\left(c_{2}\right)=\left(s_{4} s_{5}\right), m\left(c_{3}\right)=\left(s_{2} s_{3}\right)$ and, for each student $s, m(s)=\arg \max P(s)$ on $\{c \in C$ s.t. $s \in m(c)\} \cup$ $\{s\}$. In a similar way, we can support the colleges' optimal stable matching $\mu^{C}$ in which $\mu^{C}\left(c_{1}\right)=s_{1}, \mu^{C}\left(c_{2}\right)=\left(s_{2} s_{3}\right)$ and $\mu^{S}\left(c_{3}\right)=\left(s_{4} s_{5}\right)$ by a SSPE described by strategies $m\left(c_{1}\right)=s_{1}, m\left(c_{2}\right)=\left(s_{2} s_{3}\right), m\left(c_{3}\right)=\left(s_{4} s_{5}\right)$ and, for each student $s, m(s)=\arg \max P(s)$ on $\{c \in C$ s.t. $s \in m(c)\} \cup\{s\}$.

\section{FINAL REMARKS}

This paper introduces two mechanisms implementing the core correspondence of matching markets. The results solve two essential questions. First, the core of such games can be implemented in Subgame Perfect Equilibrium. And second, it provides simple mechanisms for implementing such a solution concept.

The first mechanism that we introduce implements a particular selection of the core, namely the students' optimal stable matching. Thus, this paper also provides a positive answer to the implementability of a selection of the core in matching markets. Notice that Kara and Sönmez (1997) prove that no selection of the core can be implemented in Nash Equilibrium.

Unfortunately a symmetric result cannot be provided for the set of colleges. This result points out (as Roth (1985) did) the asymmetry holding among both sides of the market. Moreover, we can also state, in the words 
of Roth, that "the college admissions problem is not equivalent to the marriage problem." Note that, in the particular case of marriage markets (colleges have only one position each), a symmetrical result for Theorem 4.1 can be stated by exchanging the role of students and colleges.

To conclude, we want to mention that Alcalde, et al. (1998) analyzed two mechanisms for job matching markets that were inspired by the mechanisms used in this paper. They show that, under some conditions, the results presented in this paper can be extended to the case in which monetary transfers play an essential role. Nevertheless, as the reader can see, the mathematical tools employed in the two papers are very different. So, even if the results of the two papers have interpretative similarities, neither can be considered a particular case of the other.

\section{REFERENCES}

Alcalde, J. (1996). "Implementation of Stable Solutions to the Marriage Problem," J. Econom. Theory 69, 240-254.

Alcalde, J., and Barberà, S. (1994). "Top Dominance and the Possibility of Strategy-proof Stable Solutions to Matching Problems," Econom. Theory 4, 417-435.

Alcalde, J., Pérez-Castrillo, D., and Romero-Medina, A. (1998). "Hiring procedures to implement stable allocations," J. Econom. Theory 82, 469-480.

Gale, D., and Shapley L. S. (1962). "College Admissions and the Stability of Marriage," Am. Math. Monthly 69, 9-15.

Jackson, M. O. (1992). "Implementation in Undominated Strategies: A Look at Bounded Mechanisms," Rev. Econom. Stud. 59, 757-775.

Kara, T., Sönmez, T. (1997) "Implementation of College Admission Rules," Econom. Theory 9, 197-218.

Romero-Medina, A. (1998). "Implementation of Stable Solutions in a Restricted Matching Market.” Rev. Econom. Design 3, 137-147.

Roth, A. E. (1982). "The Economics of Matching: Stability and Incentives," Math. Oper. Res. 7, 617-628.

Roth, A. E. (1985). "The College Admissions Problem Is Not Equivalent to the Marriage Problem," J. Econom. Theory 36, 277-288.

Roth, A. E., and Sotomayor, M. (1990). Two-Sided Matching: A Study in Game-Theoretic Modeling and Analysis. New York: Cambridge Univ. Press. 